\title{
AN ERGODIC THEOREM FOR NONCOMMUTATIVE OPERATORS
}

\author{
TAKESHI YOSHIMOTO
}

\begin{abstract}
This note contains a proof of a weighted convergence theorem which includes the ordinary "noncommuting" ergodic theorem for noncommutative families of linear contraction operators.
\end{abstract}

Given a $\sigma$-finite measure space $(X, \Re, \mu)$ we denote by $L_{p}(\mu)=L_{p}(X, \Re, \mu)$, $1 \leqq p \leqq \infty$, the usual Banach spaces. Let $\left\{w_{k}^{(j)}, k=1,2, \ldots\right\}, 1 \leqq j \leqq N$, be sequences of nonnegative numbers whose sum is one, let $\left\{u_{k}^{(j)}, k=0\right.$, $1 \cdots\}, 1 \leqq j \leqq N$, be the sequence given by

$$
u_{0}^{(j)}=1, \quad u_{k}^{(j)}=w_{1}^{(j)} u_{k-1}^{(j)}+\cdots+w_{k}^{(j)} u_{0}^{(j)}, \quad k \geqq 1,
$$

and let the sequence $\left\{\beta_{k}^{(j)}, k=1,2, \ldots\right\}, 1 \leqq j \leqq N$, be defined as follows: $\beta_{1}^{(j)}=w_{1}^{(j)}\left(=u_{1}^{(j)}\right)$, and for $k \geqq 2$,

$$
\beta_{k}^{(j)}= \begin{cases}w^{(j)} /\left(1-w_{1}^{(j)}-\cdots-w_{k-1}^{(j)}\right) & \text { if } w_{1}^{(j)}+\cdots+w_{k-1}^{(j)}<1, \\ 0 & \text { if } w_{1}^{(j)}+\cdots+w_{k-1}^{(j)}=1 .\end{cases}
$$

It is then clear that $0 \leqq u_{k}^{(j)} \leqq 1(k \geqq 0), 0 \leqq \beta_{k}^{(j)} \leqq 1(k \geqq 1)$ for $1 \leqq j$ $\leqq N$. For notational convenience, we write

$$
\begin{aligned}
& \xi\left(k_{1}, \ldots, k_{N}\right)=u_{k_{1}}^{(1)} \times \cdots \times u_{k_{N}}^{(N)}, \\
& \eta\left(k_{1}, \ldots, k_{N}\right)=\exp \left\{i \beta\left(k_{1}+\cdots+k_{N}\right)\right\} \quad(\beta: \text { a real number }), \\
& \zeta\left(k_{1}, \ldots, k_{N}\right)=\xi\left(k_{1}, \ldots, k_{N}\right) \times \eta\left(k_{1}, \ldots, k_{N}\right) .
\end{aligned}
$$

The result to be obtained below takes its rise from the question raised by $\mathrm{H}$. E. Robbins. As early as 1951 N. Dunford [5] and A. Zygmund [10], as an affirmative answer to this question, obtained some interesting individual ergodic theorems for noncommutative families of measure preserving flows with discrete and continuous parameters, respectively. Then later (1956) N. Dunford and J. T. Schwartz [6] generalized these results to those at the operator theoretic level. In this note we show that a more general approach of generalizing them can be adopted than the one used by the above authors. As far as the present author is concerned, the considerations of weighted averages

Received by the editors March 14, 1975 and, in revised form, May 12, 1975.

AMS (MOS) subject classifications (1970). Primary 47A35.

Key words and phrases. Noncommuting ergodic theorem, weighted averages, weighted convergence theorem, contraction operators, Zygmund class, Banach's convergence theorem, free direct product, Akcoglu and Chacon's convexity theorem. 
are motivated by a paper of D. L. Hanson and G. Pledger [7] who investigate conditions on the sequence $\left\{a_{i j}, i, j=0,1, \ldots\right\}$ of nonnegative numbers with $\sum_{j=0}^{\infty} a_{i j}=1$ for all $i$, such that the sequence $\sum_{j=0}^{\infty} a_{i j} f \circ \varphi^{j}$ converges (as $i \rightarrow \infty)$ in the norm of $L_{2}(\mu)$ for any $f \in L_{2}(\mu)$ and a $\mu$-measure preserving transformation $\varphi$ on $X$. (Cf. Baxter [2], [3].) However, it is easily seen that the argument used by them applies to a sequence $\left\{a_{i j}, i, j=0,1, \ldots\right\}$ of nonnegative numbers such that $\lim _{i \rightarrow \infty} \sum_{j=0}^{\infty} a_{i j}=1$ and $\sum_{j=0}^{\infty} a_{i j} \leqq K<\infty$ uniformly in $i$ for a positive constant $K$. Now take $\left\{u_{k}, k=0,1, \ldots\right\}$ to be a sequence defined as before by the requirement that $\lim _{j \rightarrow \infty} u_{j}=1$, and define the sequence $\left\{a_{i j}, i, j=0,1, \ldots\right\}$ by

$$
a_{i j}= \begin{cases}u_{j} /(i+1) & \text { for } 0 \leqq j \leqq i, \\ 0 & \text { for } j>i .\end{cases}
$$

Then it follows immediately that

$$
\begin{array}{rlr}
a_{i j} & \geqq 0 & \text { for all } i \text { and } j, \\
\sum_{j=0}^{\infty} a_{i j} & \leqq 1 & \text { uniformly in } i, \\
\lim _{i \rightarrow \infty} \sum_{j=0}^{\infty} a_{i j} & =1 . &
\end{array}
$$

In addition

$$
\sum_{j=0}^{\infty} a_{i j} f \circ \varphi^{j}=\frac{1}{i+1} \sum_{j=0}^{i} u_{j} f \circ \varphi^{j} \quad \text { for all } i,
$$

which shows that the consideration on the system $\left\{u_{k}\right\}$ is closely related to the investigation by Hanson and Pledger. A recent paper of the author [8] generalizes and extends this sort of consideration of weighted averages in a more general situation than the one stated above. (Cf. also Yoshimoto [9].) Indeed our conditions on the $a_{i j}$ 's are stronger than the ones of Hanson and Pledger, but the results obtained are much deeper than those due to them. In fact, the sequence $\sum_{j=0}^{\infty} a_{i j} f \circ \varphi^{j}$ converges (as $i \rightarrow \infty$ ) almost everywhere on $X$ for any $f \in L_{p}(\mu)$ with $1 \leqq p<\infty$ and also in the mean of order $p$ for any $f \in L_{p}(\mu)$ with $1<p<\infty$. This note generalizes the situation just described to the case of noncommutative families of linear contraction operators. The first result we shall demonstrate is the following

THEOREM 1. Let $T_{(j)}, 1 \leqq j \leqq N$, be linear operators in $L_{1}(\mu)$ with $\left\|T_{(j)}\right\|_{L_{1}(\mu)} \leqq 1$ and $\left\|T_{(j)}\right\|_{L_{\infty}(\mu)} \leqq 1$ for $1 \leqq j \leqq N$. Then for every $f \in L_{p}(\mu)$ with $p>1$, the multiple sequence

$$
\frac{1}{n_{1} \cdots n_{N}} \sum_{k_{1}=0}^{n_{1}-1} \cdots \sum_{k_{N}=0}^{n_{N}-1} \zeta\left(k_{1}, \ldots, k_{N}\right)\left(T_{(1)}^{k_{1}} \cdots T_{(N)}^{k_{N}} f\right)(x)
$$

is convergent (as $n_{1}, \ldots, n_{N} \rightarrow \infty$ independently) almost everywhere on $X$, as well as in the norm of $L_{p}(\mu)$. This sequence is dominated by a function in $L_{p}(\mu)$. Moreover, if the measure is finite then for any $f \in L_{1}(\mu)$ belonging to the 
Zygmund class, the multiple sequence converges almost everywhere on $X$, as well as in the mean of order one.

If in Theorem 1 one takes $w_{1}^{(j)}=1, w_{k}^{(j)}=0(k \geqq 2), 1 \leqq j \leqq N$, then the theorem reduces to the so-called discrete "noncommuting" ergodic theorem generalized by Dunford and Schwartz. Note here that the operators $T_{(1)}, \ldots$, $T_{(N)}$ appearing in Theorem 1 need not commute.

ProOF OF TheOREM 1. Let $\left(\mathbf{N}^{*}, \mathbb{Q}^{*}, \lambda^{*}\right)$ be the product space of the measure spaces $\left(\mathbf{N}, \mathbb{Q}, \lambda_{j}\right), 1 \leqq j \leqq N$, obtained by taking $\mathbf{N}$ to be the set of all positive integers, $\mathbb{Q}$ the $\sigma$-algebra of all subsets of $\mathbf{N}$, and $\lambda_{j}, 1 \leqq j \leqq N$, the measures defined on $Q$ by setting

$$
\lambda_{j}(\{1\})=1, \quad \lambda_{j}(\{k\})=1-w_{1}^{(j)}-\cdots-w_{k-1}^{(j)}, \quad k \geqq 2,1 \leqq j \leqq N .
$$

For each $j$, let $W_{(j)}$ be the transformation of $L_{1}\left(\lambda_{j}\right)$ to itself defined by left multiplication by the matrix

$$
W_{(j)}=\left[\begin{array}{ccccc}
\beta_{1}^{(j)} & \beta_{2}^{(j)} & \beta_{3}^{(j)} & \beta_{4}^{(j)} & \ldots \\
1-\beta_{1}^{(j)} & 0 & 0 & 0 & \ldots \\
0 & 1-\beta_{2}^{(j)} & 0 & 0 & \ldots \\
0 & 0 & 1-\beta_{3}^{(j)} & 0 & \ldots \\
. & . & . & . & \\
. & . & . & . & \\
. & . & . & . &
\end{array}\right]
$$

(cf. Chacon [4]). We use $W_{(j)}$ to denote the transformation and the matrix and present the elements of $L_{1}\left(\lambda_{j}\right)$ as column vectors. It follows then easily that each $W_{(j)}$ is a positive linear operator in $L_{1}\left(\lambda_{j}\right)$ whose $L_{1}\left(\lambda_{j}\right)$ and $L_{\infty}\left(\lambda_{j}\right)$ norms are less than or equal to one, and that

$$
\left(W_{(j)}^{k} \delta_{1}^{(j)}\right)(1)=u_{k}^{(j)}, \quad k \geqq 0,1 \leqq j \leqq N,
$$

where $\delta_{k}^{(j)}(i)=1$ if $k=i$, and $\delta_{k}^{(j)}(i)=0$ if $k \neq i$. Let us take $\left\{W_{\left(k_{1}, \ldots, k_{N}\right)}\right.$, $\left.k_{1}, \ldots, k_{N}=0,1, \ldots\right\}$ to be the free direct product of $\left\{W_{(1)} k_{1}, k_{1}=0,1, \ldots\right\}$, $\ldots,\left\{W_{(N)}^{k_{N}}, k_{N}=0,1, \ldots\right\}$ :

$$
W_{\left(k_{1}, \ldots, k_{N}\right)}=W_{(1)}^{k_{1}} \times \cdots \times W_{(N)}^{k_{N}},
$$

and set

$$
V_{(j)}^{k_{j}}=W_{\left(0, \ldots, 0, k_{j}, 0, \ldots, 0\right)}, \quad k_{j} \geqq 0,1 \leqq j \leqq N,
$$

and define, for each $j$, the direct product $\tau_{(j)}=V_{(j)} \times U_{(j)}$, where $U_{(j)} k_{j}$ $=\eta\left(k_{j}\right) T_{(j)}{ }^{k_{j}}$ for $k_{j} \geqq 0$. Then, putting

$$
g_{f}\left(i_{1}, \ldots, i_{N}, x\right)=\delta_{1}^{(1)}\left(i_{1}\right) \times \cdots \times \delta_{1}^{(N)}\left(i_{N}\right) \times f(x)
$$

for $f \in L_{p}(\mu)$ with $1 \leqq p<\infty$, we have 


$$
\begin{aligned}
\left(\tau_{(1)}^{k_{1}} \cdots\right. & \left.\tau_{(N)}^{k_{N}} g_{f}\right)(1, \ldots, 1, x) \\
& =\xi\left(k_{1}, \ldots, k_{N}\right)\left(U_{(1)}^{k_{1}} \cdots U_{(N)}^{k_{N}} f\right)(x) \\
& =\zeta\left(k_{1}, \ldots, k_{N}\right)\left(T_{(1)}^{k_{1}} \cdots T_{(N)}^{k_{N}} f\right)(x) .
\end{aligned}
$$

One may therefore apply the theorem of Dunford and Schwartz [6] to $\tau_{(1)}, \ldots, \tau_{(N)}$ with the functions $g_{f}$ for which $f$ belongs to $L_{p}(\mu)$ with $p>1$, to obtain the first and second assertions of Theorem 1. As for the third part, we first note that on the hypothesis of the theorem, the functions

$$
\frac{1}{n_{1} \cdots n_{N}} \sum_{k_{1}=0}^{n_{1}-1} \cdots \sum_{k_{N}=0}^{n_{N}-1}\left(\tau_{(1)}^{k_{1}} \cdots \tau_{(N)}^{k_{N}} g_{f}\right)(1, \ldots, 1, \cdot)
$$

for $f \in L_{1}(\mu)$ which belongs to the Zygmund class are, for $n_{1}, \ldots, n_{N} \geqq 1$, all dominated by a function in $L_{1}(\mu)$. In fact, by Dunford and Schwartz's lemma [6], there are positive operators $Q_{(j)}, 1 \leqq j \leqq N$, in $L_{1}(\mu)$ whose $L_{1}(\mu)$ and $L_{\infty}(\mu)$ norms do not exceed those of $T_{(j)}, 1 \leqq j \leqq N$, and which are such that

$$
\left|\left(T_{(j)}^{k} f\right)(\cdot)\right| \leqq Q_{(j)}^{k}(|f(\cdot)|), \quad k \geqq 0, f \in L_{1}(\mu), 1 \leqq j \leqq N .
$$

Thus, from this we have

$$
\begin{aligned}
\left|\frac{1}{n_{1} \cdots n_{N}} \sum_{k_{1}=0}^{n_{1}-1} \cdots \sum_{k_{N}=0}^{n_{N}-1}\left(\tau_{(1)}^{k_{1}} \cdots \tau_{(N)}^{k_{N}} g_{f}\right)(1, \ldots, 1, \cdot)\right| \\
\quad=\left|\frac{1}{n_{1} \cdots n_{N}} \sum_{k_{1}=0}^{n_{1}-1} \cdots \sum_{k_{N}=0}^{n_{N}-1} \zeta\left(k_{1}, \ldots, k_{N}\right)\left(T_{(1)}^{k_{1}} \cdots T_{(N)}^{k_{N}} f\right)(\cdot)\right| \\
\quad \leqq \frac{1}{n_{1} \cdots n_{N}} \sum_{k_{1}=0}^{n_{1}-1} \cdots \sum_{k_{N}=0}^{n_{N}-1}\left|\left(T_{(1)}^{k_{1}} \cdots T_{(N)}^{k_{N}} f\right)(\cdot)\right| \\
\quad \leqq \frac{1}{n_{1} \cdots n_{N}} \sum_{k_{1}=0}^{n_{1}-1} \cdots \sum_{k_{N}=0}^{n_{N}-1} Q_{(1)}^{k_{1}} \cdots Q_{(N)}^{k_{N}}(|f(\cdot)|),
\end{aligned}
$$

and the last term is dominated by a function in $L_{1}(\mu)$ on account of Dunford and Schwartz's theorem [6]. Now the third assertion may be deduced from a combination of Banach's convergence theorem and Lebesgue's dominated convergence theorem. This completes the proof of the theorem. Q.E.D.

In particular, applying the convexity theorem due to Akcoglu and Chacon [1], we have by the same argument as that of Theorem 1 the following

THEOREM 2. Let $T_{(j)}, 1 \leqq j \leqq N$, be positive linear operators in $L_{q}(\mu)$, $1 \leqq q \leqq q_{0}$ for some $q_{0}\left(1<q_{0}<\infty\right)$, such that $\left\|T_{(j)}\right\|_{L_{q}(\mu)} \leqq 1$ for $1 \leqq q$ $\leqq q_{0}$ and such that for some $f>0$ in $L_{1}(\mu)$,

$$
\sum_{k=0}^{\infty}\left(T_{(j)}^{k} f\right)(x)=\infty \text { a.e., } \quad 1 \leqq j \leqq N .
$$

Then all the statements corresponding to Theorem 1 hold true for the operators $T_{(1)}, \ldots, T_{(N)}$. 
The author wishes to express his thanks to Professor Chandler Davis and the referee for their kind advice.

\section{BIBLIOGRAPHY}

1. M. A. Akcoglu and R. V. Chacon, A convexity theorem for positive operators, Z. Wahrscheinlichkeitstheorie und Verw. Gebiete 3 (1965), 328-332. MR 32 \# 192.

2. G. Baxter, An ergodic theorem with weighted averages, J. Math. Mech. 13 (1964), 481-488. MR 29 \#2361.

3. - A general ergodic theorem with weighted averages, J. Math. Mech. 14 (1965), 277288. MR 30 \#4904.

4. R. V. Chacon, Ordinary means imply recurrent means, Bull. Amer. Math. Soc. 70 (1964), 796-797. MR 29 \#5977.

5. N. Dunford, An individual ergodic theorem for non-commutative transformations, Acta Sci. Math. Szeged 14 (1951), 1-4. MR 13, 49.

6. N. Dunford and J. T. Schwartz, Convergence almost everywhere of operator averages, J. Rational Mech. Anal. 5 (1956), 129-178. MR 17, 987.

7. D. L. Hanson and G. Pledger, On the mean ergodic theorem for weighted averages, $\mathrm{Z}$. Wahrscheinlichkeitstheorie und Verw. Gebiete 13 (1969), 141-149. MR 40 \#423.

8. T. Yoshimoto, Random ergodic theorem with weighted averages, $\mathrm{Z}$. Wahrscheinlichkeitstheorie und Verw. Gebiete 30 (1974), 149-165; Erratum, ibid. 31 (1975), 161.

9. - Induced contraction semigroups and random ergodic theorems, Dissertationes Math. (to appear).

10. A. Zygmund, An individual ergodic theorem for non-commutative transformations, Acta Sci. Math. Szeged 14 (1951), 103-110. MR 13, 661.

Department of Mathematics, Faculty of Engineering, Toyo University, Kawagoe, SAITAMA, JAPAN 